\title{
Morphological characterization of Arab and Oromo goats in northwestern Ethiopia: implications for community-based breeding programs
}

Oumer Sheriff ( $\square$ soumer74@yahoo.com )

Bahirdar University https://orcid.org/0000-0002-2028-5360

Kefyalew Alemayehu

Bahir Dar University

Aynalem Haile

International Center for Agricultural Research in the Dry Areas

Research

Keywords: Characterization, Ethiopia, indigenous goats, morphological traits

Posted Date: August 13th, 2020

DOI: https://doi.org/10.21203/rs.3.rs-49628/v1

License: (c) This work is licensed under a Creative Commons Attribution 4.0 International License. Read Full License 


\section{Abstract}

Background: An exploratory field research was conducted in Northwestern Ethiopia, to characterize the morphological features of Arab and Oromo goat populations as a first step to design breeding programs. Ten qualitative and nine quantitative traits were considered from 747 randomly selected goats. All data collected during the study period were analyzed using R statistical software, version 3.5.2, 2018.

Results: Plain white coat color was predominantly observed in Arab goats (33.72\%) while plain brown (deep and light) coat color was the most frequent in Oromo goats $(27.81 \%)$. The morphometric measurements indicated that Oromo goats have significantly $(P<0.001)$ higher body weight and linear body measurements than Arab goats. Positive, strong and highly significant $(P<0.001)$ correlations were obtained between body weight and most of the body measurements in both goat populations. The highest correlation coefficients of chest girth with body weight for Arab $(r=0.95)$ and Oromo $(r=0.92)$ goat populations demonstrated a strong association between these variables. Live body weight could be predicted with regression equations of $y=-33.65+0.89 x$ for Arab goats $\left(R^{2}=90\right)$ and $y=-37.55+0.94 x$ for Oromo goats $\left(R^{2}=85\right)$, where $y$ and $x$ are body weight and chest girth, respectively, in these goat types.

Conclusions: The morphological variations obtained in this study could be complemented by performance data and molecular characterization using DNA markers to guide the overall goat conservation and formulation of appropriate breeding and selection strategies.

\section{Background}

In Ethiopia, the population of goats is estimated at 30.2 million (1), of which about $99.97 \%$ belong to indigenous breeds. They are important sources of meat, milk, manure, fibers and skin and fulfill various cultural and religious functions (2). The productivity of indigenous goats is, however, very low and hence their contribution to the national economy is far below potential (3). Many interrelated factors including lack of viable and sustainable breeding programs are identified as important constraints (4). Characterization of the existing animal genetic resource is essential to design appropriate breeding programs for breeds that have not been adequately described either morphologically and/or genetically $(5 ; 6)$. Morphological characterization is important in breed identification and classification and the first step of it is assessing the variation of qualitative and quantitative traits (7). In Ethiopia, several on-farm and on-station morphological characterization of goats have been commenced since the onset of research on goats in mid$1970 \mathrm{~s}$ (8). For instance, FARM-Africa has undertaken morphological characterization of goats at national level and shown the presence of 14 different goat types (9). On the contrary, a very recent molecular characterization of Ethiopian indigenous goats revealed the existence of only seven distinct goat breeds (10).

Benishangul Gumuz region, located in northwestern Ethiopia, is home for about 411,503 heads of goats (11) and five goat ecotypes (Agew, Arab, Felata, Gumuz and Oromo) (12). Among these, the production systems and breeding practices of Agew and Gumuz goat populations are the most studied. For instance, two decades ago, (9) described qualitative and quantitative characteristics of Gumuz goats and classified them under western lowland goat breed. Later, the genetic diversity of this goat type was investigated by (2), while the same author assessed the diversity of Ethiopian indigenous goats using microsatellite DNA markers. Subsequently, $(13 ; 14)$ undertook phenotypic and molecular characterization of Gumuz and Agew goats when the authors studied six goat breeds found in Amhara region. Furthermore, (15) characterized Gumuz goats, under the general name western lowland goat, found in Metema district of Amhara region using high density SNP CHIPs array. Very recently, (10) investigated the genetic diversity of Gumuz goats found in Pawe special district of Benishangul Gumuz region while the author undertook molecular characterization of Ethiopian indigenous goat populations using Caprine SNP CHIP array. Overall, despite the presence of different goat types/breeds in Benishangul Gumuz region (12), characterization works focused mainly on Gumuz and/or Agew goat ecotypes. However, Arab and Oromo goat ecotypes, like many others, need to be better characterized.

The Arab goats are named after Arab/Berta tribe, the dominant tribe that generally owns these particular goat types. These goats are more adaptable to the semi-arid areas, trypano tolerant and considered as dual purpose (used for meat and milk production) (12). On the other hand, the Oromo goats are named after Oromo community, inhabit the sub-humid agro-ecology and known as meat type (12). Both goat ecotypes provide a range of products and functions for their owners. For example, they are sources of income, meat, skin, manure, signs of wealth status and used as a means of saving (16). Additionally, they have unique morphological features that are noticeable to any casual observer. However, they were neglected in goat research and development endeavors of the country and hence there is no empirical evidence that support this hypothesis. The limited available information about the two goat ecotypes, so far, has been based on on-farm survey and recall interviews (12). Therefore, the objective of the current study was to undertake morphological characterization of Arab and Oromo goat populations and suggest sustainable breeding program in the selected areas of Benishangul Gumuz region in northwestern Ethiopia.

\section{Materials And Methods Description of the study areas}

This study was carried out in two districts, Homosha and Bambasi, of Benishangul Gumuz region (Fig. 1) from December 2017 to April 2018 . The districts were selected purposively to characterize the targeted goat populations in two different agroecologies and production systems. The Homosha district is located in semiarid agro-ecology. It extends from $6^{\circ} 44^{\prime}$ to $6^{\circ} 84^{\prime}$ north latitude and from $37^{\circ} 92^{\prime}$ to $38^{\circ} 6^{\prime}$ east longitude (17) with an average altitude of 1,373 masl (18). The temperature ranges from $20-30^{\circ} \mathrm{C}$ and the mean annual rainfall is $700-1,200 \mathrm{~mm}$. The production systems are mixed 
crop-livestock and agro-pastoral (19). The Arab goats predominate in this district. The second study area, Bambasi district, is situated in the sub-humid agroecology and it is positioned at $9^{\circ} 45^{\prime}$ north latitude and $34^{\circ} 44^{\prime}$ east longitude with an elevation of 1,668 masl (20). The mean annual rainfall ranges from $900-1,500 \mathrm{~mm}$ and the average temperature is $28^{\circ} \mathrm{C}(21)$. A mixed crop-livestock system is the dominant production system (22). The Oromo goats are predominant in Bambasi district. Detailed description of the study areas is given in the previous work (16).

\section{Data collection}

A total of 747 goats (258 Arab and 489 Oromo goats) were sampled for both qualitative and quantitative measurements. The Arab goats were selected in Gumu-Abush, Sherkole, Tumet and Tsore-almetema villages of Homosha district whereas the Oromo goats were sampled in Bambasi 02, Mutsa 01, Shebora and Womba-selama villages of Bambasi district. The goats were classified into six age groups based on their dentition, i.e., kids (< 6 months), young (6-12 months), 1 pair of permanent incisors (1PPI) (1 year), 2PPI ( 2 years), 3PPI (3 years) and 4PPI ( $\geq 4$ years) (23). The kids and young goats were differentiated by asking the age of goats from owners while the goats in 1, 2, 3 and 4 and above years were differentiated by observing their dentition. From the total sample size, 629 (84.2\%) were female goats. The relatively high number of females could be attributed to the fact that they are normally retained in flocks by the goat keepers for reproduction while the males are more frequently put up for sale (16). Pregnant does were excluded from measurement to avoid over estimation of body weight (BW) and linear body measurements (LBMs). In the current study, quantitative traits, except BW, are generally named as LBMs.

Ten qualitative variables (coat color pattern, coat color type, head profile, horn presence, horn shape, horn orientation, ear orientation, wattle presence, ruff presence and hair type) were recorded by using the standard format adapted from the (6) breed descriptor list.

Nine morphometric measurements were also taken on each goat in the morning before they were released for grazing. The measurements were taken as described by (6). They included body weight (BW), the fasted live body weight (in kg); chest girth (CG), circumference of the body (in cm) immediately behind the shoulder blades and perpendicular to the body axis; body length (BL), horizontal distance (in $\mathrm{cm}$ ) from the point of shoulder to the pin bone; wither height $(\mathrm{WH})$, vertical height (in $\mathrm{cm}$ ) from the bottom of the front foot to the highest point of the shoulder; rump height (RH), vertical height from the bottom of the back foot to the highest point of the rump; chest width (CW), width (in cm) of the chest between the briskets; pelvic width (PW), horizontal distance (in $\mathrm{cm}$ ) between the extreme lateral points of the hook bone of the pelvis; horn length ( $\mathrm{HL}$ ), length of the horn (in $\mathrm{cm}$ ) on its exterior side from its root at the poll to the tip and ear length (EL), length (in $\mathrm{cm}$ ) of the external ear from its root on the poll to the tip. Body weight (kg) was recorded using suspended spring balance. The height measurements $(\mathrm{cm})$ were taken using a graduated measuring stick while the length, width and circumference measurements $(\mathrm{cm})$ were measured with plastic measuring tape. All measurements were taken after restraining and holding the goats in their natural position and before they were released for grazing.

\section{Statistical analyses}

All the data collected during the study period were encoded and fed into MS-Excel (2010). Based on the nature of data, different types of statistical analyses were used. The qualitative data were analyzed using 'datasets package version 3.5.2' of R software (24). Chi-square $\left(\chi^{2}\right)$ test was carried out when required to assess the statistical significance among qualitative variables.

The quantitative data were analyzed using 'LSM (least squares mean) package version 3.5.2' of R (24). Tukey's comparison test was used to compare the sub factors that brought significant differences.

The statistical model used was:

$Y i j k=\mu+A i+G j+(A \times G) i j+e i j k$

Where, Yijk = the values of BW and LBMs in the $i$ th age, $j$ th goat population; $\mu=$ overall mean; $A \mathrm{i}=$ fixed effect of $i$ th age $(i=<6$ months, $6-12$ months, $1 \mathrm{PPI}, 2 \mathrm{PPI}$, 3PPI and $\geq 4 \mathrm{PPI}) ; \mathrm{G}$ = fixed effect of $j$ th goat population ( $j=$ Arab, Oromo); $(\mathrm{A} \times \mathrm{G}) i j=$ interaction effect of age with goat population; and eijk $=$ effect of random residual error. Due to the fact that only a few male goats at older age classes (3PPI and $\geq 4 \mathrm{PPI}$ ) were available in the study areas, male animals were excluded in the model in the analysis of BW and LBMs.

Pearson's correlation coefficient ( $r$ ) values for the goat populations were also computed to assess the relationship between BW and LBM using 'cor.test function' of R (24). In addition, to estimate BW from LBMs, the maximum adjusted $\mathrm{R}^{2}$ method of same software was used and stepwise regression procedures were implemented to screen out the best fitted model using the 'MASS package version 7.3-51.1' of R (24). Furthermore, the 'model $r$ package version 0.1.4' was used to calculate the MSE (mean square of error). In the first step, all LBMs were entered together into the model for each goat population. Then, a group of variables having the maximum adjusted $\mathrm{R}^{2}$ and minimum MSE were selected. In addition, Akaike's information criteria (AIC) and the Bayesian information criteria (BIC) were considered. In the second step, the variables which were selected by maximum adjusted $\mathrm{R}^{2}$ and minimum MSE were entered together into the model to find the best fitted regression equation.

$Y i=\beta 0+\beta 1 \times 1+\beta 2 \times 2+\beta 3 \times 3+\beta 4 \times 4+\beta 5 \times 5+\beta 6 \times 6+\beta 7 \times 7+\beta 8 \times 8+e i$

Where, $Y i=$ dependent variable (BW); $\beta 0=$ intercept; $X 1, \ldots, X 8=$ independent variables (CG, $\mathrm{BL}, \mathrm{WH}, \mathrm{RH}, \mathrm{CD}, \mathrm{PW}, \mathrm{HL}$ and EL); $\beta 1, \ldots ., \beta 8=$ regression coefficients of the variables $X 1, \ldots, X 8$; and $e i=$ residual random error. 


\section{Results}

\section{Qualitative characteristics}

The frequency and percent of qualitative characteristics observed in male and female goats of the two populations are presented in Tables 1 and 2. The results of the study showed that plain, patchy and spotted coat color patterns were observed in both goat populations, with maximum incidence in Arab goats (66.28\% plain) and minimum incidence in Oromo goats ( $9.41 \%$ spotted). Thirteen types of coat colors were observed in the sample goat populations, of which white in Arab (33.72\%) and brown in Oromo (27.81\%) goat populations were the most frequently observed coat colors followed by brown (16.67\%) and white (21.27\%) in Arab and Oromo goats, respectively (Fig. 2). Plain black coat color was less frequent than plain white, brown or gray ones. However, amongst the mixed goat coat colors, a mixture of white and black with white dominant is the predominant coat color observed in both goat populations.

Variations between the two goat populations were also observed in other qualitative characteristics. For instance, $61.63 \%$ of Arab and $95.5 \%$ of Oromo goats were characterized by straight head. The horned goats accounted for about $97.67 \%$ and $87.93 \%$ of Arab and Oromo goats, respectively. More than two-third of Arab and almost all of Oromo goat populations have straight horn shape oriented backward in $96.51 \%$ of Arab and $85.28 \%$ of Oromo goats.

Some characteristics, such as presence of droopy/hanged down ear orientation, are also shared by roughly half of the total population in Arab goats whilst majorities (62.99\%) of Oromo goats have lateral/sideway ears. Wattles were found in Arab (14.34\%) and Oromo goats (12.07\%). It was also found that $88.76 \%$ of Arab and $86.50 \%$ of Oromo goats have no ruff. Most of Arab (90.70) and Oromo goat populations (92.23) have short and smooth hair while the rest of goats were characterized by short and course hair. In general, results of the present study showed the presence of clear morphological variations between and within Arab and Oromo goat populations. 
Table 1

Frequency $(\mathrm{N})$ and percent (in brackets) of coat color pattern and color type of goat populations by sex

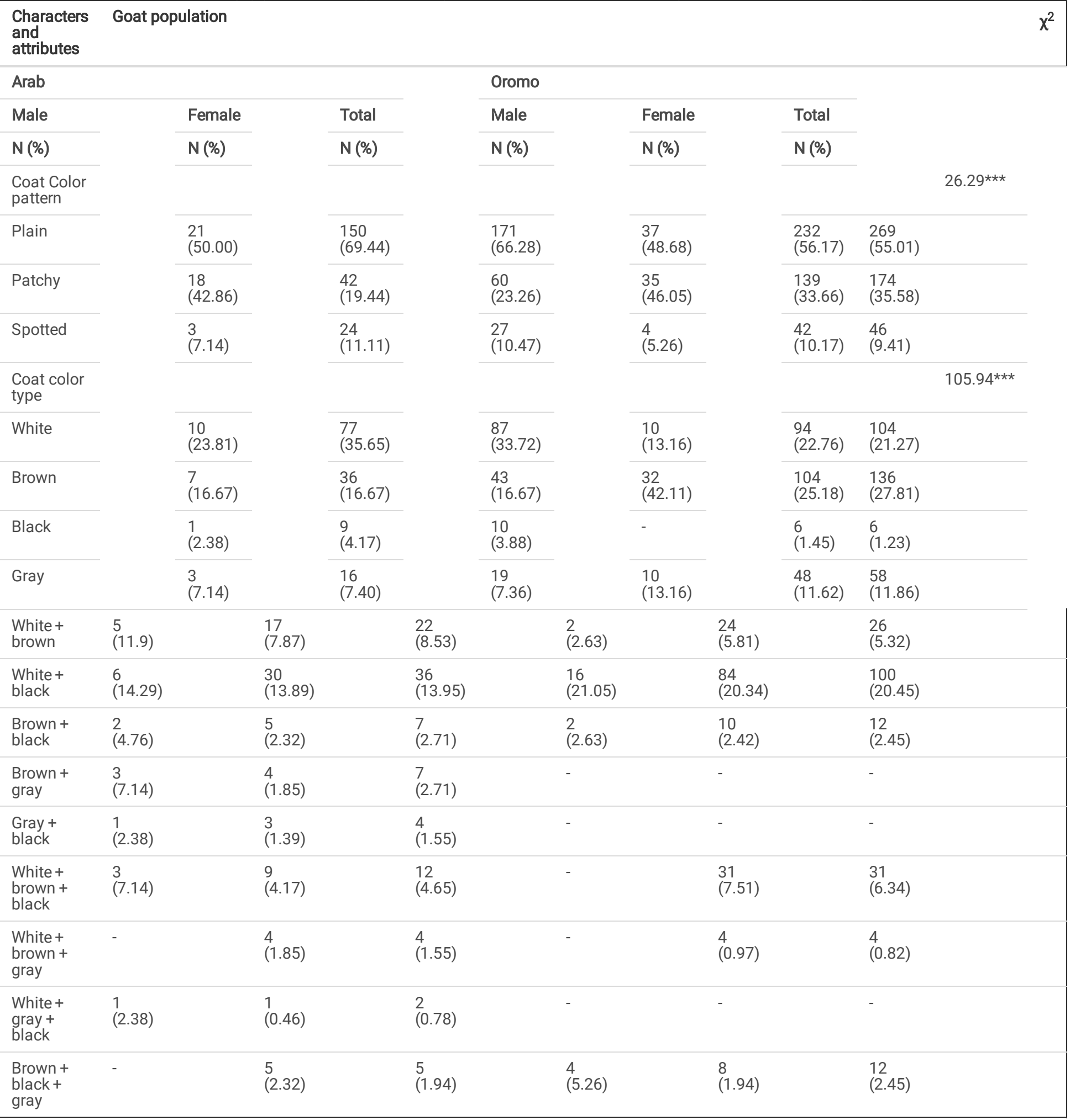

$\mathrm{N}=$ Number of goats; ${ }^{* * * \mathrm{P}} \leq 0.001$ 
Table 2

Frequency $(\mathrm{N})$ and percent (in brackets) of incidence for some qualitative features of goat populations by sex

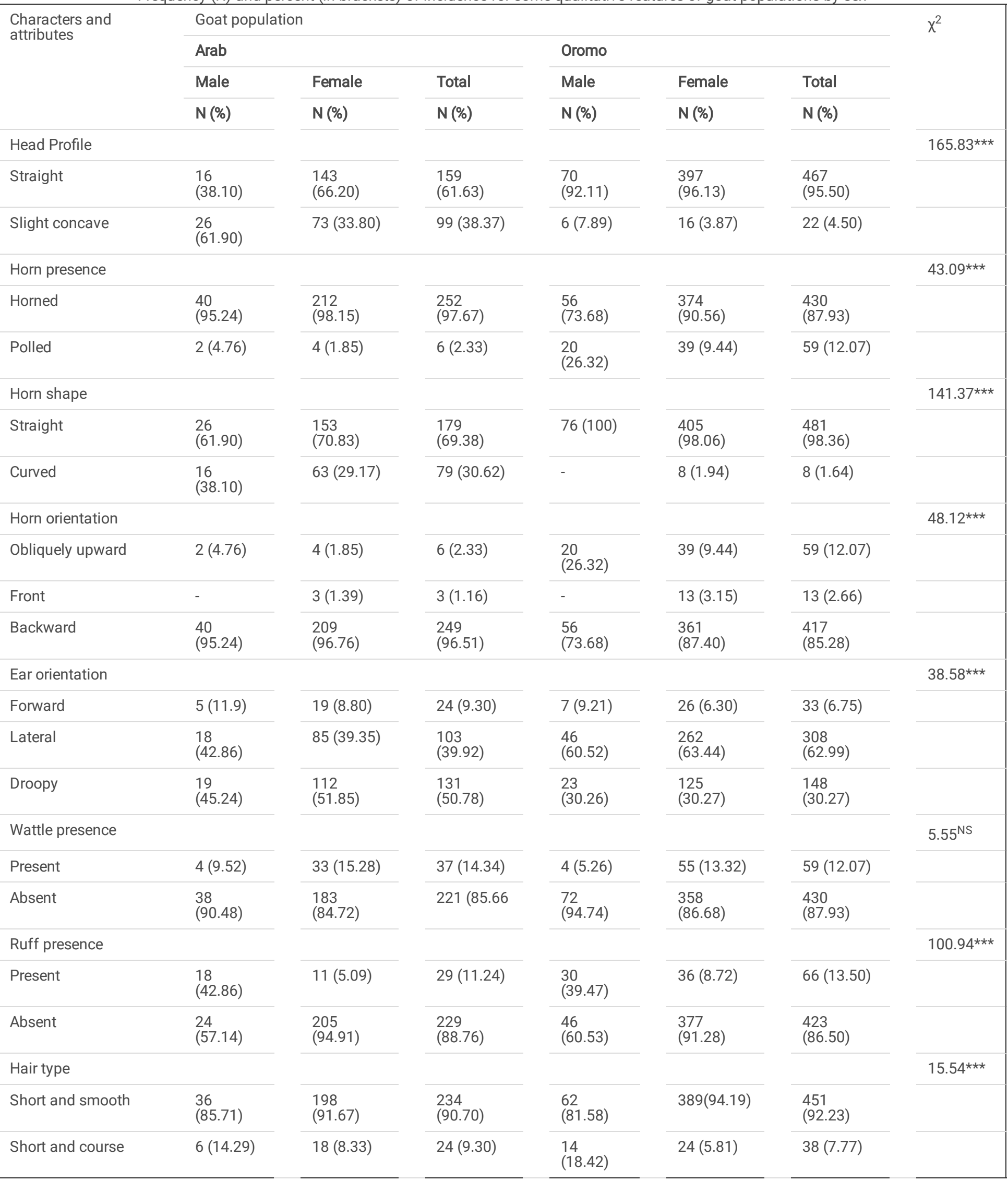

$\mathrm{N}=$ Number of goats; NS, non-significant; $* * \star \mathrm{P} \leq 0.001$ 
The least square means (LSM) and standard error (SE) for BW, CG, BL, WH, RH, CW, PW, HL and EL of female Arab goats were $22.9 \pm 0.4 \mathrm{~kg}, 63.3 \pm$ $0.4 \mathrm{~cm}, 53.5 \pm 0.4 \mathrm{~cm}, 60.1 \pm 0.4 \mathrm{~cm}, 61.5 \pm 0.3 \mathrm{~cm}, 15.6 \pm 0.1 \mathrm{~cm}, 13.6 \pm 0.1 \mathrm{~cm}, 11.0 \pm 0.2 \mathrm{~cm}$ and $13.8 \pm 0.1 \mathrm{~cm}$, respectively. The corresponding values for Oromo female goats were $26.4 \pm 0.3 \mathrm{~kg}, 67.9 \pm 0.3 \mathrm{~cm}, 57.3 \pm 0.3 \mathrm{~cm}, 64.4 \pm 0.3 \mathrm{~cm}, 66.1 \pm 0.3 \mathrm{~cm}, 16.1 \pm 0.1 \mathrm{~cm}, 13.9 \pm 0.1 \mathrm{~cm}, 11.3 \pm 0.3 \mathrm{~cm}$ and $13.9 \pm 0.1 \mathrm{~cm}$, respectively (Table 3 ). Highly significant $(p<0.001)$ differences were observed between the two goat populations in most of the studied quantitative measurements, except for horn length and ear length.

\section{Age effect}

The LSM for BW and LBMs were significantly $(\mathrm{p}<0.01)$ influenced by age groups. Except for age classes of 2PPI and 3PPI, there were significant increases in BW and other LBMs as the age increased from the youngest (kid) to the oldest (4PPI) age group (Table 3). Results clearly indicate that BW and LBMs increase proportionately with the advancement of age. This situation is however expected since the size and shape of animals change as the age increases. Maximum gain on BW and LBMs was observed between 6 to 12 months of age.

\section{Interaction effect}

The interaction effect of age with goat type significantly $(p<0.001)$ affected BW, CG, BL, WH, RH and CW, but it affected PW and HL moderately $(p<$ $0.05)$. However, there was no significant $(p>0.05)$ age by goat type interaction effect on EL. When same age class Arab and Oromo goats were compared to each other, Oromo goats had significantly $(p<0.001)$ higher measurements in most of the variables. In general, 4PPI Oromo goats had the highest measurements; whereas Arab kids had the lowest measurement in all variables. 
Table 3

Least squares means and standard error for body quantitative traits for different goat population and age groups

\begin{tabular}{|c|c|c|c|c|c|c|c|c|c|c|}
\hline Effects and & $\mathbf{N}$ & BW (kg) & CG (cm) & $\mathrm{BL}(\mathrm{cm})$ & WH (cm) & $\mathrm{RH}(\mathrm{cm})$ & $\mathrm{CW}(\mathrm{cm})$ & PW (cm) & $\mathrm{HL}(\mathrm{cm})$ & EL (cm) \\
\hline & & $\mathrm{LSM} \pm \mathrm{SE}$ & $\mathrm{LSM} \pm \mathrm{SE}$ & $\mathrm{LSM} \pm \mathrm{SE}$ & $\mathrm{LSM} \pm \mathrm{SE}$ & $\mathrm{LSM} \pm \mathrm{SE}$ & $\mathrm{LSM} \pm \mathrm{SE}$ & $\mathrm{LSM} \pm \mathrm{SE}$ & $\mathrm{LSM} \pm \mathrm{SE}$ & $\mathrm{LSM} \pm \mathrm{SE}$ \\
\hline Overall & & $24.7 \pm 0.2$ & $65.4 \pm 0.3$ & $55.5 \pm 0.4$ & $61.8 \pm 0.4$ & $63.5 \pm 0.5$ & $15.9 \pm 0.1$ & $13.9 \pm 0.1$ & $11.3 \pm 0.4$ & $13.9 \pm 0.1$ \\
\hline CV percent & & 22.69 & 9.10 & 10.66 & 9.50 & 9.65 & 6.58 & 6.05 & 23.72 & 5.60 \\
\hline Goat type & & $\star \star \star *$ & 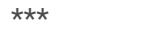 & $\star \star \star *$ & $\star \star \star *$ & $\star \star \star \star$ & 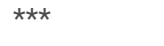 & $\star \star \star *$ & NS & NS \\
\hline Arab & 216 & $22.9 \pm 0.4$ & $63.3 \pm 0.4$ & $53.5 \pm 0.4$ & $60.1 \pm 0.4$ & $61.5 \pm 0.4$ & $15.6 \pm 0.1$ & $13.6 \pm 0.1$ & $11.0 \pm 0.2$ & $13.8 \pm 0.1$ \\
\hline Oromo & 413 & $26.4 \pm 0.3$ & $67.9 \pm 0.3$ & $57.3 \pm 0.3$ & $64.4 \pm 0.3$ & $66.1 \pm 0.3$ & $16.1 \pm 0.1$ & $13.9 \pm 0.1$ & $11.3 \pm 0.1$ & $13.9 \pm 0.1$ \\
\hline Age & & 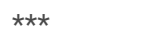 & $\star \star \star$ & $\star \star \star *$ & $\star \star \star$ & $\star \star \star$ & $\star \star \star$ & $\star \star \star *$ & $\star \star \star *$ & $\star * *$ \\
\hline Kids & 88 & $\begin{array}{l}11.6 \pm \\
0.3^{a}\end{array}$ & $\begin{array}{l}51.6 \pm \\
0.4^{a}\end{array}$ & $\begin{array}{l}42.0 \pm \\
0.5^{a}\end{array}$ & $\begin{array}{l}48.4 \pm \\
0.4^{\mathrm{a}}\end{array}$ & $\begin{array}{l}49.8 \pm \\
0.5^{a}\end{array}$ & $14.5 \pm 0.1^{\mathrm{a}}$ & $12.6 \pm 0.1^{\mathrm{a}}$ & $8.6 \pm 0.3^{a}$ & $\begin{array}{l}12.9 \pm \\
0.1^{a}\end{array}$ \\
\hline Young & 126 & $\begin{array}{l}19.6 \pm \\
0.2^{b}\end{array}$ & $\begin{array}{l}62.2 \pm \\
0.3^{b}\end{array}$ & $\begin{array}{l}52.4 \pm \\
0.3^{b}\end{array}$ & $\begin{array}{l}59.3 \pm \\
0.3^{b}\end{array}$ & $\begin{array}{l}60.9 \pm \\
0.3^{b}\end{array}$ & $15.6 \pm 0.1^{b}$ & $13.3 \pm 0.1^{\mathrm{b}}$ & $9.8 \pm 0.2^{b}$ & $\begin{array}{l}13.2 \pm \\
0.1^{\mathrm{a}}\end{array}$ \\
\hline 1PPI & 50 & $\begin{array}{l}25.8 \pm \\
0.2^{c}\end{array}$ & $\begin{array}{l}66.5 \pm \\
0.2^{c}\end{array}$ & $\begin{array}{l}56.2 \pm \\
0.3^{c}\end{array}$ & $\begin{array}{l}63.3^{ \pm} \\
0.2^{c}\end{array}$ & $\begin{array}{l}64.8 \pm \\
0.3^{c}\end{array}$ & $15.7 \pm 0.1^{b}$ & $13.7 \pm 0.1^{c}$ & $\begin{array}{l}11.3^{ \pm} \\
0.2^{c}\end{array}$ & $\begin{array}{l}13.7^{ \pm} \\
0.1^{b}\end{array}$ \\
\hline 2PPI & 63 & $\begin{array}{l}28.1 \pm \\
0.2^{d}\end{array}$ & $\begin{array}{l}69.8 \pm \\
0.2^{d}\end{array}$ & $\begin{array}{l}58.6 \pm \\
0.2^{d}\end{array}$ & $\begin{array}{l}66.4 \pm \\
0.2^{d}\end{array}$ & $\begin{array}{l}67.9 \pm \\
0.2^{d}\end{array}$ & $16.2 \pm 0.1^{c}$ & $14.2 \pm 0.1^{d}$ & $\begin{array}{l}12.0 \pm \\
0.2^{d}\end{array}$ & $\begin{array}{l}14.1^{\mathrm{I}} \pm \\
0.1^{\mathrm{b}}\end{array}$ \\
\hline 3PPI & 82 & $\begin{array}{l}30.7 \pm \\
0.2^{\mathrm{e}}\end{array}$ & $\begin{array}{l}70.9 \pm \\
0.3^{\text {de }}\end{array}$ & $\begin{array}{l}60.8 \pm \\
0.3^{\mathrm{e}}\end{array}$ & $\begin{array}{l}66.5 \pm \\
0.3^{\text {de }}\end{array}$ & $\begin{array}{l}68.5 \pm \\
0.4^{\mathrm{de}}\end{array}$ & $16.7 \pm 0.1^{d}$ & $14.4 \pm 0.1^{d}$ & $\begin{array}{l}12.1 \pm \\
0.3^{d}\end{array}$ & $\begin{array}{l}14.6 \pm \\
0.1^{d}\end{array}$ \\
\hline 4PPI & 220 & $\begin{array}{l}32.8 \pm \\
0.4^{f}\end{array}$ & $\begin{array}{l}72.4 \pm \\
0.7^{\mathrm{e}}\end{array}$ & $\begin{array}{l}63.3 \pm \\
0.7^{f}\end{array}$ & $67.9 \pm 0.7^{f}$ & $70.3 \pm 0.8^{f}$ & $16.9 \pm 0.2^{d}$ & $15.1 \pm 0.1^{\mathrm{e}}$ & $\begin{array}{l}13.8 \pm \\
0.5^{\mathrm{e}}\end{array}$ & $\begin{array}{l}15.5^{ \pm} \\
0.1^{\mathrm{e}}\end{array}$ \\
\hline Age x Goat type & & $\star \star * *$ & $\star \star * *$ & $\star \star \star *$ & $\star \star \star *$ & $\star * *$ & $\star \star \star$ & * & * & NS \\
\hline Kid $\times$ Arab & 30 & $9.4 \pm 0.2^{a}$ & $\begin{array}{l}49.4 \pm \\
0.3^{a}\end{array}$ & $\begin{array}{l}40.0 \pm \\
0.5^{\mathrm{a}}\end{array}$ & $\begin{array}{l}46.2 \pm \\
0.5^{\mathrm{a}}\end{array}$ & $\begin{array}{l}48.4 \pm \\
0.5^{\mathrm{a}}\end{array}$ & $14.0 \pm 0.2^{\mathrm{a}}$ & $12.4 \pm 0.1^{\mathrm{a}}$ & $8.8 \pm 0.4^{b}$ & $12.8 \pm 0.1$ \\
\hline Kid $x$ Oromo & 58 & $\begin{array}{l}14.4 \pm \\
0.2^{b}\end{array}$ & $\begin{array}{l}54.3 \pm \\
0.4^{b}\end{array}$ & $\begin{array}{l}44.4 \pm \\
0.5^{\mathrm{b}}\end{array}$ & $\begin{array}{l}50.9 \pm \\
0.5^{\mathrm{b}}\end{array}$ & $\begin{array}{l}51.6 \pm \\
0.6^{\mathrm{b}}\end{array}$ & $15.1 \pm 0.2^{b}$ & $12.8 \pm 0.1^{b}$ & $8.4 \pm 0.5^{a}$ & $13.0 \pm 0.1$ \\
\hline Young x Arab & 43 & $\begin{array}{l}16.1 \pm \\
0.2^{c}\end{array}$ & $\begin{array}{l}56.2 \pm \\
0.3^{c}\end{array}$ & $\begin{array}{l}46.8 \pm \\
0.5^{c}\end{array}$ & $\begin{array}{l}53.4 \pm \\
0.4^{c}\end{array}$ & $\begin{array}{l}54.2 \pm \\
0.5^{c}\end{array}$ & $15.2 \pm 0.1^{b}$ & $13.0 \pm 0.1^{b}$ & $\begin{array}{l}10.3 \pm \\
0.4^{d}\end{array}$ & $12.9 \pm 0.1$ \\
\hline Young x Oromo & 82 & $\begin{array}{l}21.1^{ \pm} \\
0.1^{d}\end{array}$ & $\begin{array}{l}64.8 \pm \\
0.2^{d}\end{array}$ & $\begin{array}{l}54.8 \pm \\
0.3^{d}\end{array}$ & $\begin{array}{l}61.9 \pm \\
0.3^{d}\end{array}$ & $\begin{array}{l}63.8 \pm \\
0.3^{e}\end{array}$ & $\begin{array}{l}15.7 \pm \\
0.1^{\mathrm{cd}}\end{array}$ & $13.5 \pm 0.1^{c}$ & $9.5 \pm 0.3^{c}$ & $13.3 \pm 0.1$ \\
\hline 1PPI x Arab & 17 & $\begin{array}{l}22.8 \pm \\
0.2^{\mathrm{e}}\end{array}$ & $\begin{array}{l}63.9 \pm \\
0.3^{d}\end{array}$ & $\begin{array}{l}53.9 \pm \\
0.4^{d}\end{array}$ & $\begin{array}{l}61.8 \pm \\
0.4^{d}\end{array}$ & $\begin{array}{l}62.4 \pm \\
0.5^{d}\end{array}$ & $15.5 \pm 0.1^{c}$ & $13.5 \pm 0.1^{c}$ & $\begin{array}{l}10.4 \pm \\
0.4^{d}\end{array}$ & $13.7 \pm 0.1$ \\
\hline 1PPI x Oromo & 33 & $\begin{array}{l}26.6 \pm \\
0.1^{f}\end{array}$ & $\begin{array}{l}67.2 \pm \\
0.2^{\mathrm{e}}\end{array}$ & $\begin{array}{l}56.8 \pm \\
0.2^{\mathrm{e}}\end{array}$ & $\begin{array}{l}63.7 \pm \\
0.2^{\mathrm{e}}\end{array}$ & $\begin{array}{l}65.4 \pm \\
0.2^{\mathrm{ef}}\end{array}$ & $15.8 \pm 0.1^{d}$ & $\begin{array}{l}13.7 \pm \\
0.1^{\mathrm{cd}}\end{array}$ & $\begin{array}{l}11.5 \pm \\
0.2^{\mathrm{e}}\end{array}$ & $13.7 \pm 0.1$ \\
\hline $2 \mathrm{PPI} \times$ Arab & 22 & $\begin{array}{l}25.9 \pm \\
0.1^{f}\end{array}$ & $\begin{array}{l}66.5 \pm \\
0.3^{e}\end{array}$ & $\begin{array}{l}56.3 \pm \\
0.4^{\mathrm{e}}\end{array}$ & $\begin{array}{l}63.8 \pm \\
0.4^{\mathrm{e}}\end{array}$ & $\begin{array}{l}64.9 \pm \\
0.4^{\mathrm{e}}\end{array}$ & $15.8 \pm 0.1^{d}$ & $13.9 \pm 0.1^{d}$ & $\begin{array}{l}11.8 \pm \\
0.4^{\mathrm{e}}\end{array}$ & $14.0 \pm 0.1$ \\
\hline 2PPI x Oromo & 41 & $\begin{array}{l}28.7 \pm \\
0.1^{\mathrm{g}}\end{array}$ & $\begin{array}{l}70.7 \pm \\
0.1^{\mathrm{fg}}\end{array}$ & $\begin{array}{l}59.2 \pm \\
0.2^{f}\end{array}$ & $\begin{array}{l}67.0 \pm \\
0.2^{\mathrm{g}}\end{array}$ & $\begin{array}{l}68.7 \pm \\
0.2^{h}\end{array}$ & $16.3 \pm 0.1^{\mathrm{e}}$ & $14.3 \pm 0.1^{\mathrm{e}}$ & $\begin{array}{l}12.1 \pm \\
0.2^{f}\end{array}$ & $14.1 \pm 0.1$ \\
\hline 3PPI x Arab & 28 & $\begin{array}{l}29.3 \pm \\
0.1^{\mathrm{g}}\end{array}$ & $69.7 \pm 0.2^{f}$ & $\begin{array}{l}59.7 \pm \\
0.3^{f}\end{array}$ & $65.4 \pm 0.3^{f}$ & $\begin{array}{l}67.4 \pm \\
0.4^{\mathrm{g}}\end{array}$ & $16.2 \pm 0.1^{\mathrm{e}}$ & $14.3 \pm 0.1^{\mathrm{e}}$ & $\begin{array}{l}11.9 \pm \\
0.3^{\mathrm{ef}}\end{array}$ & $14.5 \pm 0.1$ \\
\hline 3PPI x Oromo & 54 & $\begin{array}{l}32.8 \pm \\
0.2^{\mathrm{h}}\end{array}$ & $\begin{array}{l}72.7 \pm \\
0.3^{h}\end{array}$ & $\begin{array}{l}62.5 \pm \\
0.4^{\mathrm{g}}\end{array}$ & $\begin{array}{l}68.3 \pm \\
0.4^{\mathrm{h}}\end{array}$ & $70.2 \pm 0.5^{i}$ & $\begin{array}{l}17.4 \pm \\
0.1^{\mathrm{g}}\end{array}$ & $14.3 \pm 0.1^{\mathrm{e}}$ & $\begin{array}{l}12.3 \pm \\
0.4^{f}\end{array}$ & $14.7 \pm 0.1$ \\
\hline 4PPI x Arab & 76 & $\begin{array}{l}31.7 \pm \\
0.2^{h}\end{array}$ & $\begin{array}{l}71.1 \pm \\
0.5^{\mathrm{g}}\end{array}$ & $\begin{array}{l}61.6 \pm \\
0.7^{\mathrm{g}}\end{array}$ & $\begin{array}{l}66.7 \pm \\
0.6^{g}\end{array}$ & $\begin{array}{l}69.3 \pm \\
0.7^{\mathrm{hi}}\end{array}$ & $16.6 \pm 0.2^{f}$ & $14.8 \pm 0.1^{f}$ & $\begin{array}{l}13.3^{ \pm} \\
0.6^{\mathrm{g}}\end{array}$ & $15.3 \pm 0.1$ \\
\hline 4PPI x Oromo & 145 & $37.0 \pm 0.5^{\mathrm{i}}$ & $77.0 \pm 0.9^{i}$ & $\begin{array}{l}69.5 \pm \\
1.3^{h}\end{array}$ & $72.2 \pm 1.2^{\mathrm{i}}$ & $74.0 \pm 1.4^{j}$ & $18.2 \pm 0.4^{\mathrm{h}}$ & $\begin{array}{l}16.2 \pm \\
0.3^{g}\end{array}$ & $\begin{array}{l}15.8 \pm \\
1.2^{\mathrm{h}}\end{array}$ & $16.0 \pm 0.3$ \\
\hline
\end{tabular}

Column means within each sub-class with different superscript letter are statistically different. NS, non-significant; *p $\leq 0.05 ; * * \star p \leq 0.001 ; B W$, body weight; CG, chest girth; BL, body length; WH, wither height; RH, rump height; CW, chest width; PW, pelvic width; HL, horn length; EL, ear length; PPI = pair 


\section{Correlation between BW and LBMs}

In both Arab and Oromo goat populations, BW had positive and highly significant $(p<0.001)$ correlations with all LBMs except with EL in the Arab goats, in which the correlation was non-significant ( $p>0.05)$ (Table 4). Furthermore, most of the quantitative traits in both goat populations showed positive and highly significant $(P<0.001)$ associations with one another. In Arab goats, BW had strong positive correlation with CG $(r=0.95)$, BL $(r=$ 0.93), $\mathrm{WH}(r=0.89), \mathrm{RH}(r=0.89), \mathrm{PW}(r=0.75)$ and CW ( $r=0.65)$. Similarly, in the Oromo goats, BW had strong positive correlation with CG ( $r=0.92)$, $\mathrm{WH}(r=0.83), \mathrm{BL}(r=0.82), \mathrm{RH}(r=0.81)$ and $\mathrm{PW}(r=0.58)$. The strongest positive and highly significant correlation between BW and CG suggested that CG could provide the best estimate in predicting live BW of goats in the study areas.

Table 4

Coefficient of correlation between body weight and linear body measurements (above diagonal for Arab and below diagonal for Oromo goat populations)

\begin{tabular}{|c|c|c|c|c|c|c|c|c|c|}
\hline & BW & CG & BL & WH & RH & $\mathrm{CW}$ & PW & $\mathrm{HL}$ & EL \\
\hline BW & & $0.95^{\star \star \star}$ & $0.93^{* * *}$ & $0.89 * \star \star$ & $0.89 * * *$ & $0.65^{\star \star \star}$ & $0.75^{\star \star \star}$ & $0.38 * \star \star$ & $0.10^{\mathrm{NS}}$ \\
\hline CG & $0.92^{\star \star \star}$ & & $0.88^{\star \star \star}$ & $0.86^{\star \star \star}$ & $0.86^{\star \star \star}$ & 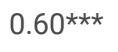 & $0.70^{\star \star \star \star}$ & $0.36^{\star \star \star}$ & $0.05^{\mathrm{NS}}$ \\
\hline $\mathrm{BL}$ & $0.82^{\star \star \star}$ & $0.87 * \star \star$ & & $0.86^{\star \star \star *}$ & $0.86 * \star \star *$ & $0.62^{\star \star \star}$ & $0.69 * \star \star$ & $0.31 * \star \star$ & $0.13^{\star}$ \\
\hline WH & $0.83^{\star \star \star}$ & $0.90^{\star \star \star}$ & $0.78^{\star \star \star}$ & & $0.85^{\star \star \star}$ & $0.62^{\star \star \star}$ & $0.67 * \star \star *$ & $0.32^{\star \star \star}$ & $0.03^{\mathrm{NS}}$ \\
\hline $\mathrm{RH}$ & $0.81^{\star \star \star *}$ & $0.89 * \star \star$ & $0.77^{\star \star \star}$ & $0.85^{\star \star \star}$ & & 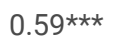 & $0.65^{\star \star \star}$ & $0.24^{\star \star \star *}$ & $0.11^{\mathrm{NS}}$ \\
\hline$C D$ & $0.44^{\star \star \star}$ & $0.42^{\star \star \star}$ & 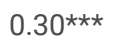 & $0.39 * \star \star$ & $0.40 * \star \star *$ & & $0.81^{\star \star \star}$ & $0.21^{\star \star \star}$ & $0.13^{\mathrm{NS}}$ \\
\hline PW & $0.58 * \star \star$ & $0.56^{\star \star \star \star}$ & $0.53^{\star \star \star}$ & $0.52^{\star \star \star}$ & $0.51^{\star \star \star}$ & $0.76^{\star \star \star}$ & & $0.27 * \star \star *$ & $0.16^{*}$ \\
\hline $\mathrm{HL}$ & $0.40 * * *$ & $0.37^{\star \star \star}$ & $0.31^{\star \star \star}$ & $0.32^{\star \star \star}$ & $0.35^{\star \star \star}$ & $0.19 * \star \star$ & $0.25^{\star \star \star}$ & & $-0.29 * \star \star$ \\
\hline EL & $0.35^{\star \star \star}$ & $0.32^{\star \star \star}$ & $0.25^{\star \star \star}$ & $0.29 * \star \star$ & $0.28 * \star \star$ & $0.15^{\star \star \star}$ & $0.20 * \star \star *$ & $0.12^{*}$ & \\
\hline
\end{tabular}

$N S$, non-significant; ${ }^{*} p \leq 0.05 ;{ }^{* *} p \leq 0.001 ; B W$, body weight; $C G$, chest girth; BL, body length; WH, wither height; RH, rump height; CW, chest width; PW, pelvic width; HL, horn length; EL, ear length; PPI = pair of permanent incisors; $S E$, standard error

\section{Prediction of BW from LBMs}

Equations predicting BW from LBM of Arab and Oromo goats are presented in Table 5. In the prediction of BW, the multiple stepwise regressions found seven parameters (CG, BL, RH, PW, HL, WH and EL) to be significant $(p<0.05)$ for Arab goats and five parameters $(C G, E L, C W, B L$ and $H L)$ to be significant $(p<0.05)$ for Oromo goats. In the present study, high coefficient of determination $\left(R^{2}\right)$ that ranged between $90-96 \%$ in Arab goats and $85-$ $86 \%$ in Oromo goats and low residual mean square (MSE) values between 2.11 to 5.00 in Arab goats and 2.72 to 3.04 in Oromo goats were recorded using the regression analyses. 
Table 5

Multiple regression analysis of live BW on different body measurements of Arab and Oromo goats in all age groups

\begin{tabular}{|c|c|c|c|c|c|c|c|c|c|c|c|c|c|}
\hline \multirow[t]{2}{*}{ Breed } & \multirow[t]{2}{*}{ Model } & \multicolumn{8}{|c|}{ Parameters } & \multirow{2}{*}{$\begin{array}{l}\text { Adj. } \\
\mathrm{R}^{2}\end{array}$} & \multirow[t]{2}{*}{ AIC } & \multirow[t]{2}{*}{$\mathrm{BIC}$} & \multirow[t]{2}{*}{ MSE } \\
\hline & & $\beta 0$ & $\beta 1$ & $\beta 2$ & $\beta 3$ & $\beta 4$ & $\beta 5$ & $\beta 6$ & $\beta 7$ & & & & \\
\hline \multirow[t]{7}{*}{ Arab } & CG & -33.65 & 0.89 & & & & & & & 0.90 & 922.15 & 932.14 & 5.00 \\
\hline & $\mathrm{CG}+\mathrm{BL}$ & -33.09 & 0.54 & 0.41 & & & & & & 0.94 & 809.18 & 822.49 & 2.86 \\
\hline & $\mathrm{CG}+\mathrm{BL}+\mathrm{RH}$ & -34.03 & 0.47 & 0.34 & 0.14 & & & & & 0.95 & 791.72 & 808.36 & 2.60 \\
\hline & $\mathrm{CG}+\mathrm{BL}+\mathrm{RH}+\mathrm{PW}$ & -40.16 & 0.44 & 0.32 & 0.14 & 0.73 & & & & 0.95 & 776.47 & 796.43 & 2.39 \\
\hline & $\mathrm{CG}+\mathrm{BL}+\mathrm{RH}+\mathrm{PW}+\mathrm{HL}$ & -40.74 & 0.41 & 0.31 & 0.16 & 0.70 & 0.18 & & & 0.96 & 764.88 & 788.18 & 2.24 \\
\hline & $\mathrm{CG}+\mathrm{BL}+\mathrm{RH}+\mathrm{PW}+\mathrm{HL}+\mathrm{WH}$ & -40.84 & 0.39 & 0.28 & 0.13 & 0.66 & 0.17 & 0.09 & & 0.96 & 758.55 & 785.17 & 2.15 \\
\hline & $\begin{array}{l}\mathrm{CG}+\mathrm{BL}+\mathrm{RH}+\mathrm{PW}+\mathrm{HL}+\mathrm{WH}+ \\
\mathrm{EL}\end{array}$ & -42.13 & 0.39 & 0.27 & 0.12 & 0.60 & 0.21 & 0.10 & 0.12 & 0.96 & 756.51 & 786.46 & 2.11 \\
\hline \multirow[t]{5}{*}{ Oromo } & CG & -37.55 & 0.94 & & & & & & & 0.85 & 1676.19 & 1688.34 & 3.04 \\
\hline & $C G+E L$ & -42.52 & 0.92 & 0.47 & & & & & & 0.85 & 1665.31 & 1681.50 & 2.95 \\
\hline & $C G+E L+C W$ & -45.32 & 0.89 & 0.46 & 0.30 & & & & & 0.86 & 1656.16 & 1676.39 & 2.87 \\
\hline & $\mathrm{CG}+\mathrm{EL}+\mathrm{CW}+\mathrm{BL}$ & -45.41 & 0.77 & 0.48 & 0.35 & 0.13 & & & & 0.86 & 1645.29 & 1669.57 & 2.78 \\
\hline & $\mathrm{CG}+\mathrm{EL}+\mathrm{CW}+\mathrm{BL}+\mathrm{HL}$ & -44.76 & 0.74 & 0.48 & 0.34 & 0.13 & 0.09 & & & 0.86 & 1637.51 & 1665.84 & 2.72 \\
\hline
\end{tabular}

$C G$, chest girth; $B L$, body length; $R H$, rump height; $P W$, pelvic width; $H L$, horn length; WH, wither height; $E L$, ear length and $C W$, chest width; $R^{2}$, coefficient of determination; AIC, Alkaike's information criteria; SBC (BIC), Bayesian information criteria and MSE, mean square error

\section{Discussion}

The current study was exclusively based on qualitative records and quantitative measurements to characterize and identify the morphological features of Arab and Oromo goat populations of Benishangul Gumuz region in northwestern Ethiopia.

Based on our results on the analysis of qualitative variables, the two goat populations have various coat color patterns and wide range of coat color types. This may be attributed to lack of systematic selection and would offer an opportunity for setting-up selection or breeding program in the study areas. However, the predominant coat colors and their patterns should be well included when goat improvement programs are designed. In addition, most of the qualitative traits were not influenced by sex except horn absence and ruff presence which were mostly expressed in male goats. The higher proportion of polled males than females in both goat populations could be due to either goat keepers' high preference to polled male goats or high frequency of short-horned allele (HoP) in males. The present result agrees with (25) who reported higher number of polled males than females in western Ethiopian goats. However, horn presence is an advantage for thermoregulation $(26 ; 27)$ and better reproductive performance (28; 29$)$. Likewise, the presence of wattle is associated with thermoregulatory function, milk yield and reproductive performance such as higher prolificacy, litter size, fertility and conception rate (27) and higher body measurements (30). Therefore, the occurrence of horn and wattle would be used as selection criteria by goat keepers of the study areas for improved performance.

(31) reported that on the basis of wither height; adult goats can be classified as large (>65 cm), small to medium (51-65 cm) and dwarf (<50 cm). According to the current results, both Arab and Oromo goats can be grouped under small to medium sized breeds. However, Oromo goats had significantly higher averages of BW and LBMs, showing that animals belonging to this population have better body conformation for meat production than animals in Arab goat population. Such difference was also observed in a study conducted by (12) on the same goat types in northwestern Ethiopia. The variation between these goat populations could arise due to difference in genetic characteristics and/or environmental conditions that may affect phenotypic variance (32). The relatively harsher environment, in terms of feed and water shortage and high environmental temperature, under which the Arab goats are raised could have been largely responsible for their lower body dimensions. Because these stress factors could have prevented the Arab goats from expressing their genetic potential. This is in line with what has been reported by (33). The authors documented that temporal and spatial variation in weather, plant productivity and subsequent nutrition could have major impacts on physical growth of animals. However, at the scope of the present study, it is difficult to associate body size, shape and conformation to any genetic background. Future studies could look in to genetic differences of the two goat populations. The coefficients of variation (CV) obtained for quantitative traits such as BW, CG, BL, $\mathrm{WH}, \mathrm{RH}$ and $\mathrm{HL}$ were relatively higher. This could be due to absence of selection, or the body parts are affected more by the environment than others (34).

Investigation on the effect of age on quantitative measurements of goats indicated that BW and LBMs increase proportionately with the advancement of age. Similar findings were reported by (15) for western lowland goats and (25) for goats found in western Ethiopia. However, the maximum body gain was observed between 6 months to 12 months. This may be due to natural increase in dry matter intake after the goats reached age at puberty. 
(35) also found maximum body gain at 9 months of age for Rohilkhand goats in India. The current average body measurements of goats in different age classes are higher than previous findings from similar age western lowland goats reported by (15).

The correlations among quantitative traits were generally positive and highly significant that ranged from $13-95 \%$ in Arab goats and $12-92 \%$ in Oromo goats with the highest correlation between BW and CG in both goat populations ( $r=0.95$ in Arab goats and 0.92 in Oromo goats). In agreement with this finding, several authors $(13 ; 36 ; 37 ; 25 ; 38)$ reported the highest correlation between BW and CG for some Ethiopian goats. This specifies that CG could be the best trait in predicting BW and may be used as a selection criterion in the study areas.

The positive and significant correlations among quantitative traits obtained in this study indicated that both goat populations have harmonious body conformation, reflecting balanced physical growth. Furthermore, it shows that the traits are under the same genetic influence due to additive genetic effect (39). This implies selection for one or more of these traits may consistently increase other traits that have positive association with the selected trait/s. In addition, selection of positively correlated traits would have paramount importance in designing breeding programs in that the selection will lead to significant improvement of body weight and other body measurements that are of economic importance (34).

The regression analyses of BW with LBMs in the present study identified seven traits in Arab and five traits in Oromo goats to predict BW. Similarly, (25) found seven traits in female $\left(R^{2}=83 \%\right)$ and five traits in male $\left(R^{2}=88 \%\right)$ to predict BW of western Ethiopian goats. (37) also reported five traits to estimate BW of Woyto-Guji and Central Highland goats with $\mathrm{R}^{2}$ values of $84 \%$ and $79 \%$, respectively. Nevertheless, CG was found to be the most appropriate variable to explain more variation in both Arab (adjusted $\mathrm{R}^{2}=90 \%$ ) and Oromo goat populations (adjusted $\mathrm{R}^{2}=85 \%$ ). The high coefficients of determination $\left(R^{2}\right)$ in the current study indicated the strong association and success of LBMs in describing more variation in BW. This may be helpful for local goat keepers to make selection and cull decisions as it can be relatively low cost, high accuracy and consistency (40). Body measurements in some cases can be more reliable than modern weighing machines as the latter can give biased results caused by gut fullness (41). As shown in Table 5, addition of a new variable to the model did not always increase the adjusted $\mathrm{R}^{2}$. However, AIC, BIC and MSE decreased as a new variable was added with little or no influence on the adjusted $\mathrm{R}^{2}$. On the other hand, addition of unnecessary variables to the model may increase the error. Therefore, addition of other LBMs to CG did not result in significant increase in adjusted $\mathrm{R}^{2}$, though it improved the accuracy of prediction by decreasing the error.

In the study areas, where formal breed data recording schemes are not well established (16) and goat keepers could not easily access weighing scales and understand complex formula, BW could be predicted from the regression equation $y=-33.65+0.89 x$ for Arab goats and $y=-37.55+0.94 x$ for Oromo goats, where $y$ and $x$ are BW and CG measurements, respectively. These formulas indicate that an increase of one cm of CG would result in an increase of 0.89 and $0.94 \mathrm{~kg}$ of BW in Arab and Oromo goat populations, respectively, which is comparable with the findings on goats elsewhere in Ethiopia $(13 ; 25 ; 37)$. The possibility of using simple body measurements that can easily be measured in the field to predict important economic traits have been demonstrated by $(42 ; 43)$. Overall, assessment of BW and LBMs in both goat populations based on the expressed regression equations remains very important for avoiding errors of visual determination of animal weights in the study areas where weighing balance cannot be easily accessed.

\section{Conclusions}

The morphological characterization of Arab and Oromo goats found in Benishangul Gumuz region of northwestern Ethiopia demonstrated phenotypic variations between the two goat populations. In addition, the goats in the study areas had shown different phenotypic characteristics with relative to western lowland goat breed. However, it would be useful to include performance data into characterization studies to understand which genotype has comparative advantage within an agroecological zone. Furthermore, an investigation on the molecular characterization using molecular markers like SNP will complement the results obtained from morphometric differentiation and also be helpful in high resolution characterization, conservation and formulation of breeding and selection strategies.

\section{Abbreviations}

AIC: Akaike's information criteria; BIC: Bayesian information criteria; BL: Body length; BW: Body weight; BoARD: Bureau of agriculture and rural development; CG: Chest girth; cm: Centimeter; CSA: Central statistical agency; CV: Coefficient of variation; CW: Chest width, DNA: Deoxyribonucleic acid; EL: Ear length; FAO: Food and agricultural organization; HL: Horn length; ICARDA: International center for agricultural research in the dry areas; ILRI: International livestock research institute; kg: Kilogram; LBMs: Linear body measurements; LSM: Least square mean; masl: meter above sea level; MSE: Mean square of error; mm: millimeter; PPI: Pair of permanent incisors; PW: Pelvic width; RH: Rump height; SE: Standard error; SNP: Single nucleotide polymorphism; WH: Wither height.

\section{Declarations}

\section{Availability of data and materials}

All data generated or analyzed during the current study are available from the corresponding author on reasonable request. 


\section{Ethics approval and consent to participate}

Not applicable. However, the current study and the proposed parent study were approved by Bahir Dar University College of Agriculture and Environmental Studies (BDUCAES) and Bahir Dar University Biotechnology Research Institute (BDUBRI). Following endorsement by the BDUCAES and BDUBRI, Assosa University (AsU) was informed about the objectives of the study through a support letter (Ref. 1/2241/134 dated back to November 13, 2017) from BDUCAES. After reviewing the proposal, AsU wrote a permission and support letter to agricultural and rural development offices of Bambasi and Homosha districts. Then, the corresponding author of this paper and four development agents from the two districts selected the goat owners for the present study. Finally, the goat owners were informed about the research and asked for verbal consent to confirm us to take the morphometric measurements on the selected goats. Four independent peasant association administrators acted as witnesses for voluntary informed decision making of the goat owners.

\section{Consent for publication}

Not applicable.

\section{Competing Interests}

The authors declare that they have no competing interests.

\section{Funding}

The financial support for this study was provided by Biotechnology Research Institute (BRI) of Bahir Dar University, Ethiopia.

\section{Authors' contributions}

OS performed the data collection and analysis and wrote this paper. KA and AH participated in coordination and helped to draft the manuscript. All the authors read and approved the final manuscript.

\section{Acknowledgements}

The corresponding author gratefully acknowledges the Federal Ministry of Education, Ethiopia, for the PhD fellowship award, Biotechnology Research Institute of Bahir Dar University for funding this research, farmers who allowed their goats free for inventory purpose and all experts and development agents in the study areas for their cooperation during data collection.

\section{References}

1. CSA. 2017. Federal Democratic Republic of Ethiopia Central Statistical Agency, Agricultural sample survey, Report on livestock and livestock holding characteristics. Statistical Bulletin No. 585, Vol. 2, Addis Ababa Ethiopia, April, 2017.

2. Tesfaye AT. 2004. Genetic characterization of indigenous goat populations of Ethiopia using microsatellite DNA markers. A PhD thesis submitted to the national dairy research institute, Deemed University. Karnal-Haryana, India.

3. Legese G, Fadiga M. 2014. Small ruminant value chain development in Ethiopia: Situation analysis and trends. ICARDA/ILRI Project Report. Nairobi, Kenya.

4. Kosgey IS, Okeyo AM. Genetic improvement of small ruminants in low-input, smallholder production systems: technical and infrastructural issues. Small Ruminant Research. 2007;70:76-88.

5. Mwacharo JM, Okeyo AM, Kamande GK, Rege JEO. The small East African Shorthorn zebu cows in Kenya. Linear body measurements. Trop Anim Health Prod. 2006;38:65-74.

6. FAO. 2012. Phenotypic characterization of animal genetic resources. FAO Animal Production and Health Guidelines No. 11. Rome. http://www.fao.org/docrep/015/i2686e/i2686e00 (accessed on 10-08-2018).

7. Delgado JV, Barba C, Camacho ME, Sereno FTPS, Martinez A, Vega-Pla JL. Livestock characterization in Spain. AGRI. 2001;29:7-18.

8. Aschalew T, Sisay L, Ameha S, Abebe M, Zinash S. 2000. National goat research strategy in Ethiopia. In: Markel RC, Abebe G, Goetsch AL, editors. The opportunities and challenges of enhancing goat production in East Africa: Proceeding of a conference held at Awassa, Debub University. pp. 1-5.

9. FARM Africa. Goat types of Ethiopia and Eritrea. In: Physical description and management systems. Published jointly by FARM-Africa. London: International Livestock Research Institute), Nairobi, Kenya; 1996. 
10. Mekuriaw G. 2016. Molecular characterization of Ethiopian indigenous goat populations: genetic diversity and structure, demographic dynamics and assessment of the Kisspeptin gene polymorphism. A dissertation submitted to the department of Microbial, Cellular and Molecular Biology, Addis Ababa University, Addis Ababa, Ethiopia.

11. CSA 2014. Federal Democratic Republic of Ethiopia Central Statistical Agency. Key findings of the 2013/2014 agricultural sample survey. Country Summary. Addis Ababa, Ethiopia, October, 2014.

12. Getinet A, Hegde BP, Bekele T, Enyew N, Workneh A. Phenotypic characterization of goat types in northwestern Ethiopia. Ethiopian Journal of Animal Production. 2005;5:13-32.

13. Hassen H, Michael B, Rischkowsky B, Tibbo M.. Phenotypic characterization of Ethiopian indigenous goat populations. Afr J Biotech. 2012a;11(73):13838-46.

14. Hassen H, Lababidi S, Rischkowsky B, Baum M, Tibbo M. Molecular characterization of Ethiopian indigenous goat populations. Trop Anim Health Prod. 2012b;44(6):1239-46.

15. Abegaz SG, Sölkner J, Gizaw G, Dessie T, Haile A, Wurzinger M. Description of production systems and morphological characteristics of Abergelle and Western lowland goat breeds in Ethiopia: implication for community-based breeding programmes. Animal Genetic Resources. 2013;53:69-78.

16. Oumer S, Kefyalew A, Aynalem H. Production systems and breeding practices of Arab and Oromo goat keepers in northwestern Ethiopia: implications for community-based breeding Programs. Tropical Animal Health and Production. 2019;52:1467-1478.

17. Abebe R, Wolde A. Preliminary survey on equine trypanosomiasis and its vectors in Asosa and Homosha districts in Benishangul Gumuz Regional State, northwest Ethiopia. Livest. Res. Rural Dev. 2010;22. http://www.Irrd.org//rrd22/1/abeb22018 (accessed on 02-12-2018).

18. Elevationmap.net 2018. Topographic Map of Homosha Town, Homosha, Asosa, Ethiopia. http://elevationmap.net/homosha-town-homoshaasosa-et-1011136468 (accessed on 02-12-2018).

19. Homosha BoARD. Homosha Bureau of Agriculture and Rural Development. Physical and socio-economic profile of Homosha district. Ethiopia: Unpublished report. Homosha; 2018.

20. Latitude.to. maps, geolocated articles, latitude longitude coordinate conversion 2018. GPS coordinates of Bambasi, Ethiopia. Latitude: 9.7500 Longitude: 34.7333. http://latitude.to/articles-by-country/et/ethiopia/329555/bambasi (accessed on 02-12-2018).

21. Merkorewos H. 2008. Assessment of local economic development potential and proposal of interventions for Bambasi and Bullen Weredas. Study on emerging regions development program, UNDP/UNCDF and Ministry of Federal Affairs.

22. Bambasi BoARD. Bambasi Bureau of Agriculture and Rural Development. Physical and socio-economic profile of Bambasi district. Ethiopia: Unpublished report. Bambasi; 2018.

23. FAO. 1994. A manual for the primary animal health care worker. Guidelines for training, guidelines for adaptation. Rome. http://www.fao.org/docrep/t0690e/t0690e00 (accessed on 12-10-2018).

24. R Core Team. (2018). R: A language and environment for statistical computing. R Foundation for Statistical Computing, Vienna, Austria. https://www.R-project.org/ (accessed on 26-04-2018).

25. Ahmed S, Kefelegn K, Kefena E. Morphological characterization of indigenous goats in Western Ethiopia: implication for community-based breeding programmes. Animal genetic resources. 2016;58:53-62.

26. Robertshaw D. Mechanisms for the control of respiratory evaporative heat loss in panting animals. J Appl Physiol. 2006;101:664-8.

27. Hagan JK, Apori SO, Bosompem M, Ankobea G, Mawuli A. Morphological Characteristics of indigenous goats in the coastal Savannah and forest eco-zones of Ghana. J Anim Sci Adv. 2012;2(10):813-21.

28. Al-Ghalban AM, Tabbaa MJ, Kridli RT. Factors affecting semen characteristics and scrotal circumference of Damascus bucks. Small Ruminant Research. 2004;53:141-9.

29. Kridli RT, Tabbaa MT, Sawalha RM, Amashe MG. Comparative study of scrotal circumference and semen characteristics of mountain black goat and its crossbred with Damascus goat as affected by different factors. Jordan Journal of Agricultural Science. 2005;1(1):18-25.

30. Jing L, Ren-Jun Z, Guo-Rong Z, Qing-Ran Y, Hua-Ming M. Quantitative and qualitative body traits of Longling yellow goats in China. Agricultural Sciences in China. 2010;9(3):408-15.

31. Devendra C, Bums M. 1983. Goat production in the tropics. 2nd edition. Farnham Royal, Common Wealth Agricultural Bureau. pp. 1-60.

32. Yakubu A, Salako AE, Imumorin IG, Ige AO, Akinyemi MO. (2010). Discriminant analysis of morphometric differentiation in the West African Dwarf and Red Sokoto goats. South African Journal of Animal Science. 2010;40:381-387.

33. Brown DE, Maher CR, Mitchell CD. 2006. A comparison of Pronghorn body measurements throughout western North America. Proceedings Proghorn Antelop Workshop 22.USA. pp. 125-137.

34. Mavule BS, Muchenje V, Bezuidenhout CC, Kunene NW. Morphological structure of Zulu sheep based on principal component analysis of body measurements. Small Ruminant Research. 2013;111:23-30.

35. Ahmad F, Patel BHM, Rijasnaz VV. Relationship of body weight with linear body measurements in Rohilkhand local goats. Indian Journal of Animal Research. 2013;47(6):521-6.

36. Mahilet D. 2012. Characterization of Hararghe highland goat and their production system in eastern Hararghe. Haramaya University, Ethiopia. MSc. thesis.

Page $13 / 15$ 
37. Gebreyesus G, Haile A, Dessie T. Breeding scheme based on community-based participatory analysis of local breeding practices, objectives and constraints for goats around Dire Dawa, Ethiopia. Livest. Res. Rural Dev. 2013;25. http://www.Irrd.org/Irrd25/3/grum25048 (accessed on 11-072018).

38. Zergaw N, Tadelle D, Kefelegn K. Indigenous breeding practices and selection criteria of goat owners in Konso and Meta-Robi districts, Ethiopia: implications for designing community-based breeding strategy. Livest. Res. Rural Dev. 2016;28. http://www.Irrd.org//rrd28/7/zerg28133 (accessed on 22-02-2018).

39. Jimmy S, David M, Donald KR, Dennis M. Smallholder goat breeding systems in humid, sub-humid and semi-arid agro-ecological zones of Uganda. Global Veterinaria. 2010;4(3):283-91.

40. Musa AM, Idam NZ, Elamin KM. Heart Girth Reflect Live Body Weight in Sudanese Shogur Sheep under Field Conditions. World Veterinary journal. 2012;2(4):54-6.

41. Obike OM, Ibe SN, Oke UK. Estimation of pre-and post-weaning body weight of rabbits in a humid tropical environment using linear body measurements. American-Eurasian Journal of Agricultural Environmental Science. 2010;9(4):440-4.

42. Vargas S, Larbi A, Sanchez M. Analysis of size and conformation of native Creole goat breeds and crossbreds used in smallholder agrosilvopastoral systems in Puebla, Mexico. Trop Anim Health Prod. 2007;39:279-86.

43. Ozkaya S, Bozkurt Y. The accuracy of prediction of body weight from body measurements in beef cattle. Archiv Tierzucht. 2009;52:371-7.

\section{Figures}

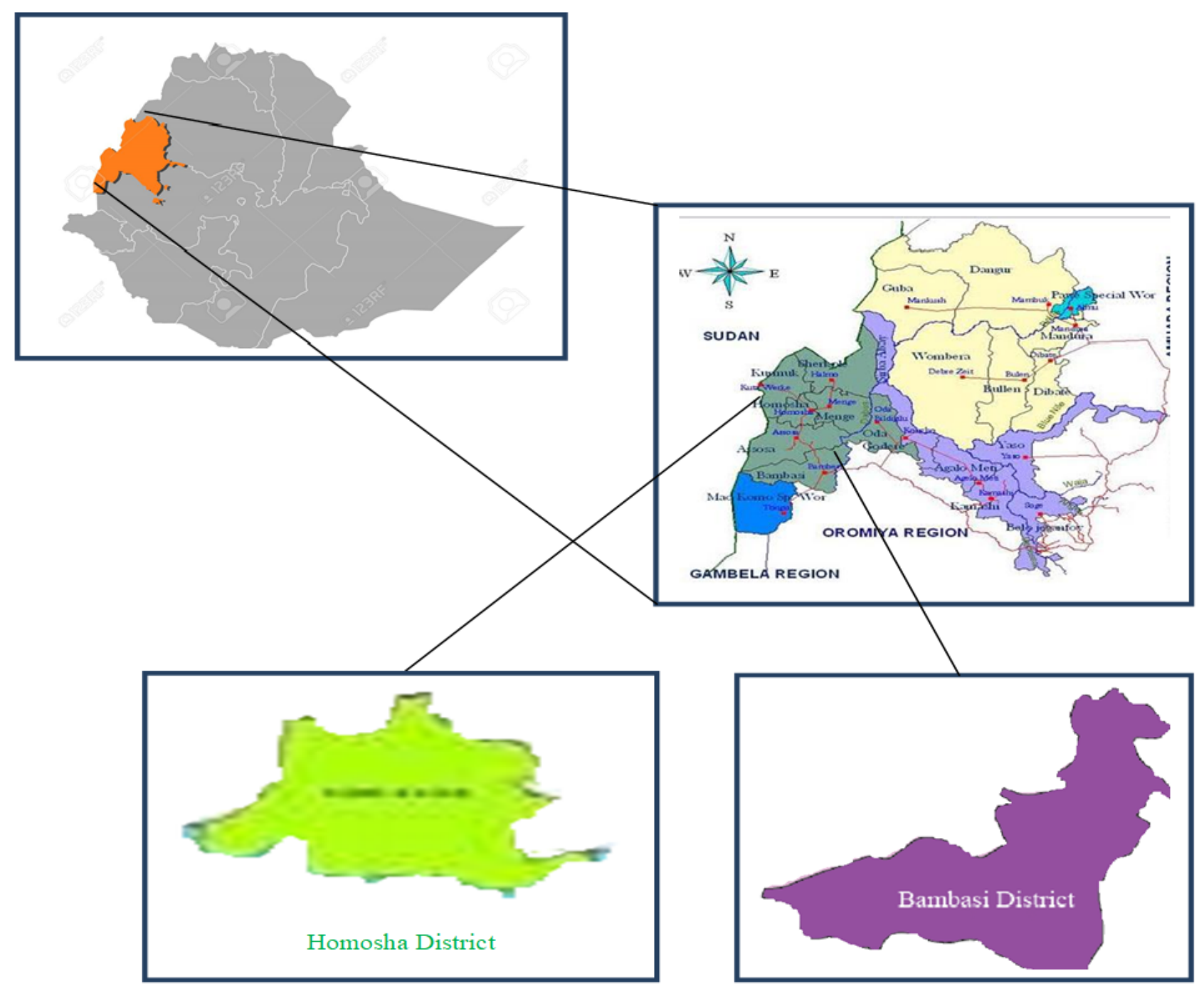

Figure 1

Map of the study areas 

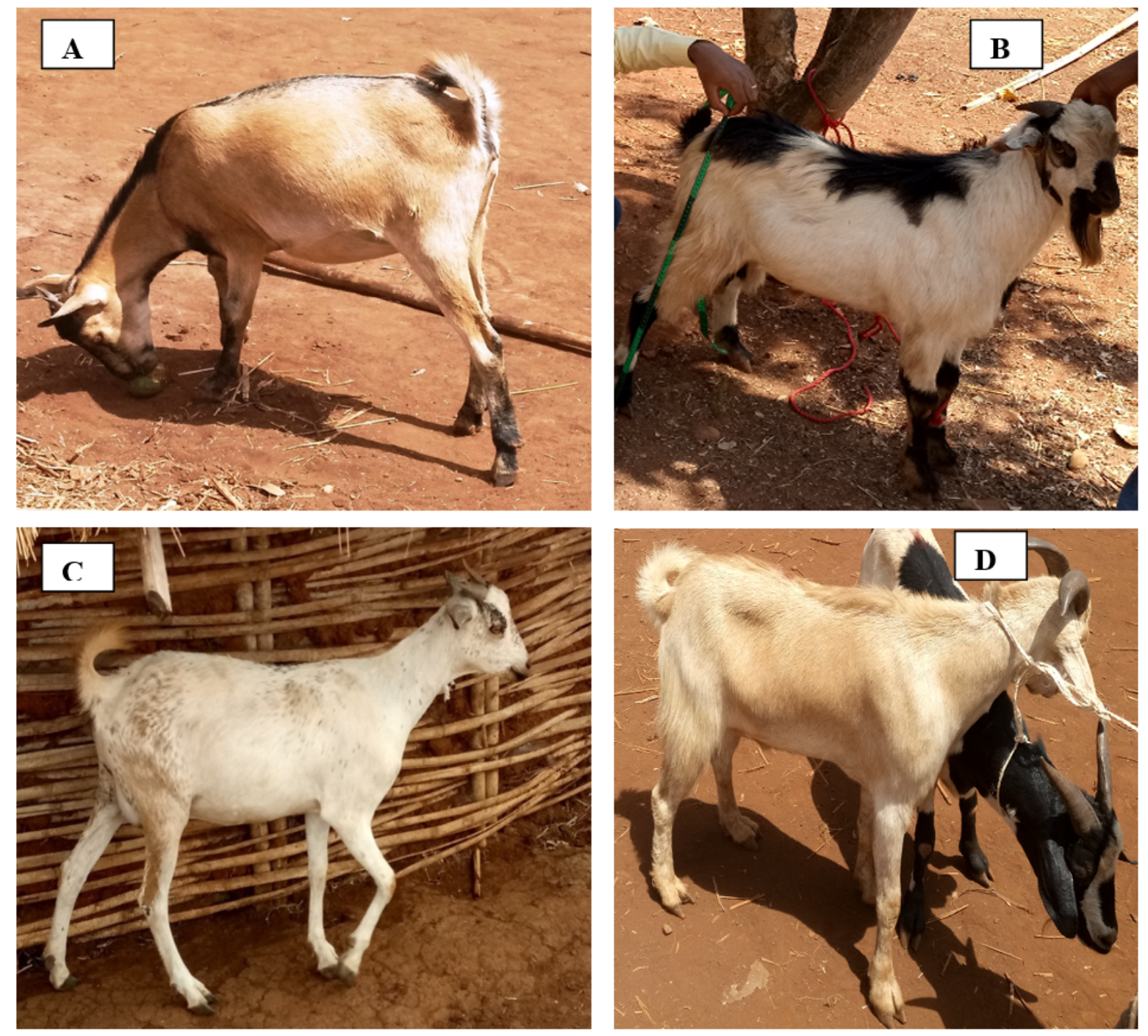

Figure 2

(a) adult Arab doe, (b) young Arab buck, (c) Adult Oromo doe and (d) Adult Oromo buck 